Scientific Visualization, 2019, volume 11, number 3, pages 76 - 87, DOI: 10.26583/sv.11.3.07

\title{
Visualization of the interaction region of an oblique shock wave with a boundary layer by the radiation of a nanosecond surface sliding discharge
}

\author{
I. Mursenkova ${ }^{1}$, A. Sazonov ${ }^{2}$, Yu. Liao ${ }^{3}$, I. Ivanov 4 \\ Lomonosov Moscow State University \\ 1 ORCID: 0000-0002-7181-4533, murs i@physics.msu.ru \\ 2 ORCID: 0000-0002-7930-9283 \\ 3 ORCID: 0000-0002-5776-1949 \\ 4 ORCID: 0000-0002-7786-6780
}

\begin{abstract}
The spatial structure of radiation of a surface sliding discharge with a duration of $\sim 300$ ns (plasma sheet) in inhomogeneous supersonic air flows including oblique shock wave in a discharge chamber of shock tube was experimentally investigated. The flows were created behind plane shock waves with Mach numbers 2.8-4.2; flow Mach numbers were 1.30-1.60. The flow in the discharge chamber included an oblique shock wave generated by the interaction of a supersonic flow with a small obstacle, and then reflected from the upper wall of the discharge chamber. In this case, the shock wave interacted with the boundary layer of the supersonic flow, which formed on the streamlined wall. A surface sliding discharge with a length of $100 \mathrm{~mm}$ and $30 \mathrm{~mm}$ wide was initiated on a streamlined surface at a certain moment in time. The discharge current, emission spectra, and spatial characteristics of the radiation under various conditions of discharge initiation were obtained and analyzed in experiments. The time dependence of geometry of the discharge glow region was obtained by digital processing of discharge images in flows. CFD simulation was performed to determine the shock-wave structure of the flow in the channel with an obstacle under experimental conditions. The comparison of experimental data with the results of numerical calculation of the supersonic flow field was done. It was shown that the spatial distribution of radiation from a surface sliding discharge visualizes the low density areas and the separation zone formed under the interaction of an oblique shock wave with a boundary layer.
\end{abstract}

Keywords: Flow vizualization by the discharge radiation, nanosecond surface sliding discharge, oblique shock wave, boundary layer, flow separation zone, cfd simulation.

\section{INTRODUCTION}

In high-speed flows in the channels the areas of interaction between the boundary layer and shock waves arise. The interaction of an inclined shock wave (oblique shock) with the boundary layer has been studied for several decades [1-6]. A strong pressure gradient caused by the impact of a shock wave on the boundary layer can lead to the flow separation. This phenomenon is accompanied by an increase in the dynamic load on the surface, its high local heating, and a rise in the drag coefficient. In addition, the interaction of the shock wave and the boundary layer can cause unsteady formation of vortices [3, 4]. In rocket propulsion systems, these effects affect the flow in the nozzles of supersonic engines [6]. In a number of experimental studies of flows of this class [3, 4] and works on numerical modeling $[1,2,6,7]$, it was found that in the separation zone the pressure and the heat transfer coefficient increase sharply. Generally, numerical simulation reflects the main features of the studied flows correctly, however the calculated values in developed separation zones generated by strong shock waves differ from experimental ones [5]. As a result, further investigation of the interaction of shock waves with boundary layers is required. 
The usage of low-temperature plasma of surface discharges of various types (plasma actuators) in plasma aerodynamics is aimed to correct the flow regime, including the reduce of dynamic and thermal loads on the surface, controlling the laminar-turbulent transition in the boundary layer, the position of separation zones and shock waves [8-10], and the processes of combustion [11]. Pulsed distributed surface sliding discharge of nanosecond duration (plasma sheet) can be used as an actuator to influence the flow [9]. It consists of channels sliding along the dielectric surface and forming a plasma layer comparable in thickness with the boundary layer of a supersonic flow in a shock tube $(\sim 0.5 \mathrm{~mm})$. Surface sliding discharge glow can visualize a laminar and turbulent boundary layer [12]. The short duration of nanosecond discharges $(\sim 100 \mathrm{~ns})$ makes it possible to use the registration of radiation to visualize the structure of unsteady supersonic plane [12] and three-dimensional flows [13]. The radiation of glow-type discharges with a duration of more than one millisecond is mainly used to visualize the structure of stationary flows $[10,14]$.

The purpose of this work was an experimental study of the spatial distribution of the glow of a pulsed surface sliding discharge in inhomogeneous supersonic flows with an oblique shock wave in the discharge chamber of the shock tube. It was important to study the fundamental physical processes under the development of a nanosecond discharge in an inhomogeneous medium, and to study the structure of the near-surface flow in the area of a pulsed energy input to develop flow control methods in such conditions. Numerical simulations of the flow fields were carried out to compare the density field with the discharge glow structure. The possibility to visualize the interaction region of the boundary layer with an oblique shock wave based on radiation registration is discussed.

\section{EXPERIMENTAL CONDITIONS}

\section{Description of the experimental setup}

The experiments were carried out using a shock tube with a low-pressure chamber with a length of $3 \mathrm{~m}$, a rectangular channel with an internal cross section of $24^{\prime} 48 \mathrm{~mm}^{2}$, and a discharge chamber of the same cross section (Fig. 1 a) [9, 12]. Supersonic air flows were created behind plane shock waves with Mach numbers 2.8-4.2. The flow velocities were 700-1150 $\mathrm{m} / \mathrm{s}$, Mach numbers of the flows were $1.30-1.60$, the density was $0.10-0.14 \mathrm{~kg} / \mathrm{m}^{3}$. Under these conditions, the duration of a uniform co-current flow behind the shock-wave front was 200-500 $\mu \mathrm{s}$, and the length of this flow behind the shock-wave front was about $30 \mathrm{~cm}$ [12]. In a shock tube the thickness of the boundary layer increases from zero at the shock wave front in the direction of the contact surface, and the boundary layer becomes turbulent at a certain distance from the shock wave front [15]. The Reynolds number of the flow was $\sim 10^{5}$, estimated from the size of the shock tube channel. The thickness of the laminar boundary layer on the channel walls did not exceed $1 \mathrm{~mm}[9,12]$.

In the discharge chamber, on the lower and upper walls, flat electrodes of surface sliding discharges $10 \mathrm{~cm}$ long with an inter-electrode distance of $3 \mathrm{~cm}$ were located. The side walls of the discharge chamber on a 17-cm-long area were quartz glasses with a transmission band of 200-2800 $\mathrm{nm}$, which allowed recording discharge radiation and spectra. The registration of the integral discharge glow was performed using photo cameras located at different angles to the camera windows. The upper plasma sheet was initiated in the experiments, its radiation was investigated in the boundary layer interacting with an oblique shock (inclined shock wave) (Fig. 1b). The discharge spectra were recorded by AvaSpec-2048FT spectrometer with wavelength range 174-1100 $\mathrm{nm}$. The fiber-optic cable (UV/VIZ, $100 \mu \mathrm{m}$ diameter) was placed at an angle to the plane of the upper plasma sheet. 


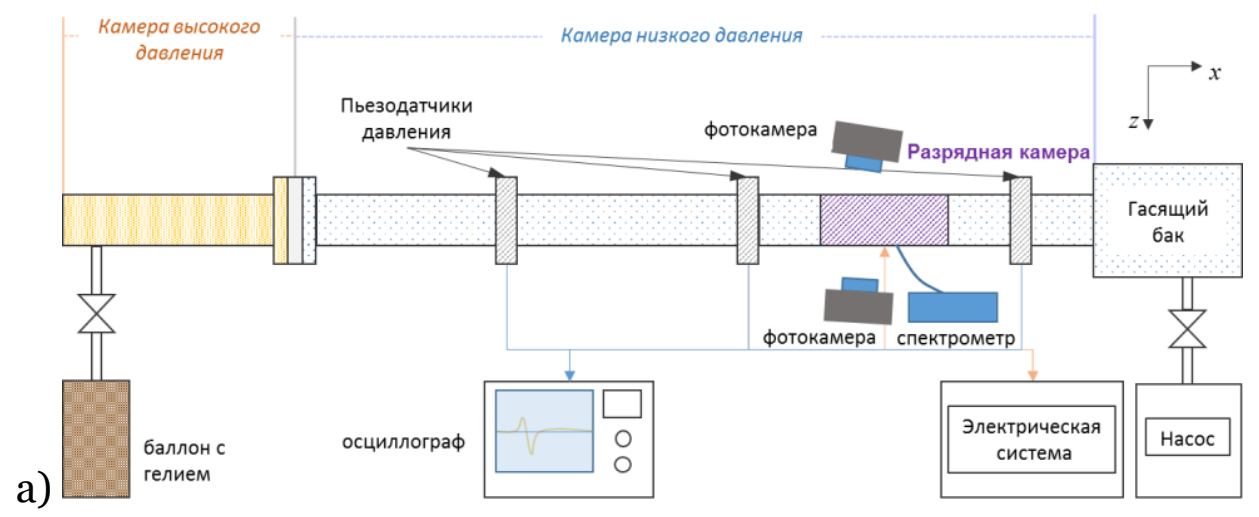

b)

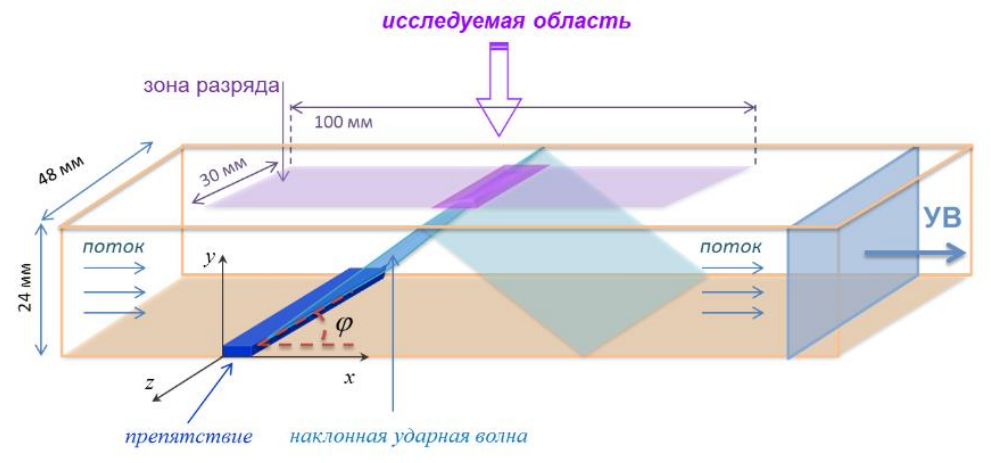

Fig. 1. a) Scheme of the shock tube and diagnostic equipment; b) the flow structure in the discharge chamber with an obstacle on the bottom wall.

\section{The structure of the gas dynamic flow in the discharge chamber}

A small dielectric obstacle in the form of a rectangular parallelepiped measuring 48.0' $6.2^{\prime} 1.9 \mathrm{~mm}^{3}$ (Fig. 1b) was placed on the bottom wall of the discharge chamber at a distance of about $1.5 \mathrm{~cm}$ from the leading edge of the lower electrode. The long part of the obstacle was perpendicular to the discharge camera glasses. After diffraction of a plane shock wave on an obstacle for $\sim 200 \mu \mathrm{s}$, supersonic flow was established by a co-current flow. The quasistationary flow field in the channel of the discharge chamber contained an oblique shock wave that interacted with the boundary layer on the upper wall of the discharge chamber (Fig. 1b). The diagrams of two types of interaction that are realized in the interaction of an oblique shock wave with a boundary layer are shown in Fig. 2. In the case of a laminar boundary layer, the interaction with the boundary layer can be continual, but contains a region of low density (Fig. 2 a); in the other case, an interaction occurs with the formation of separation zone (Fig. 2 b).

A pulsed surface sliding discharge was initiated on the upper wall of the discharge chamber in the time range of 70-1200 $\mu$ s after the shock wave passed the obstacle. The discharge triggering was synchronized with the passage of the shock wave front from the signals of the piezoelectric pressure sensors in the shock tube channel. The initiation of the discharge was carried out at a given time after the shock wave passed through the test area in the discharge chamber, including the stage of quasistationary supersonic flow around the obstacle. 


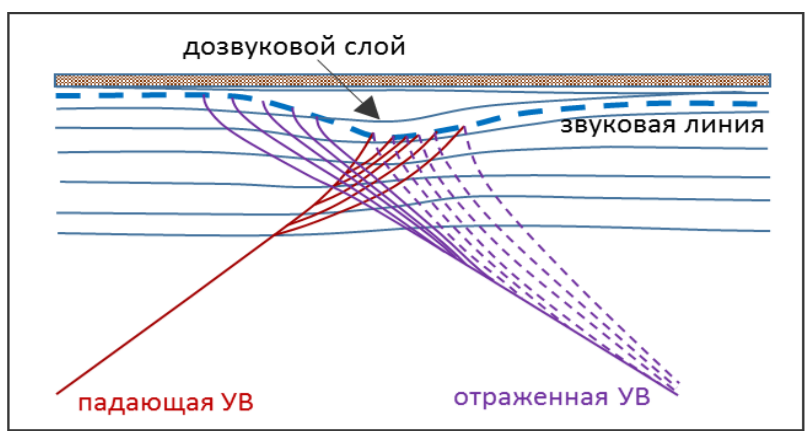

a

a)

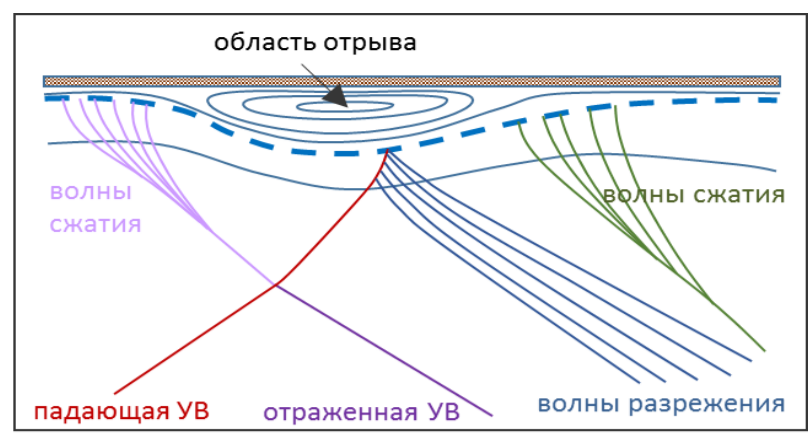

6

Fig. 2. Diagrams of the interaction of an oblique shock wave with a boundary layer: a) without separation; b) with the separation zone.

\section{The characteristics of surface sliding discharge}

A pulsed surface sliding discharge was developed in a thin gas layer near the surface of the dielectric when a pulsed voltage of $25 \mathrm{kV}$ was applied to the discharge electrodes, providing a significant specific energy input. The discharge current was recorded by a low-inductive shunt of a special design. The discharge current was $\sim 1 \mathrm{kA}$, the duration did not exceed 500 ns (Fig. 3). The main energy input to the gas occurs within 120-150 ns of the first half period of the discharge current, i.e. almost instantaneously as compared with the characteristic gasdynamic flow times in the boundary layer $[9,12]$. The electric field applied to the discharge gap was $\mathrm{E}=8.33 \mathrm{kV} / \mathrm{cm}$, and the reduced electric field $E / N$ under the experimental conditions reached $3 \cdot 10^{-19} \mathrm{~V} \cdot \mathrm{m}^{2}$, where $N$ is the concentration of molecules). Under such conditions, the processes of excitation of electronic levels of nitrogen molecules $\mathrm{N}_{2}$ and oxygen $\mathrm{O}_{2}$, ionization and dissociation of molecules $[8,16]$ actively occur in discharges in the air. This leads to the presence of significant ultraviolet part in the emission spectra due to the emission of molecular nitrogen and atomic lines (Fig. 4).

The ionization rate, on which the electron concentration in the discharge plasma and conductivity depend, is determined by the local reduced electric field $E / N$. Therefore, the density distribution in the medium affects the structure of the discharge radiation. The photographs of the discharge in still air show that the discharge consists of parallel channels of close intensity that completely fill up the discharge gap with a length of $10 \mathrm{~cm}$ (Fig. $5 \mathrm{a}, \mathrm{b}$ ). In an inhomogeneous flow with an oblique shock wave, the discharge was developed as a single intense channel (Fig. 5c), the direction of development of the channel is perpendicular to the flow velocity. The geometry of the discharge current depended on the density distribution in the discharge region. 


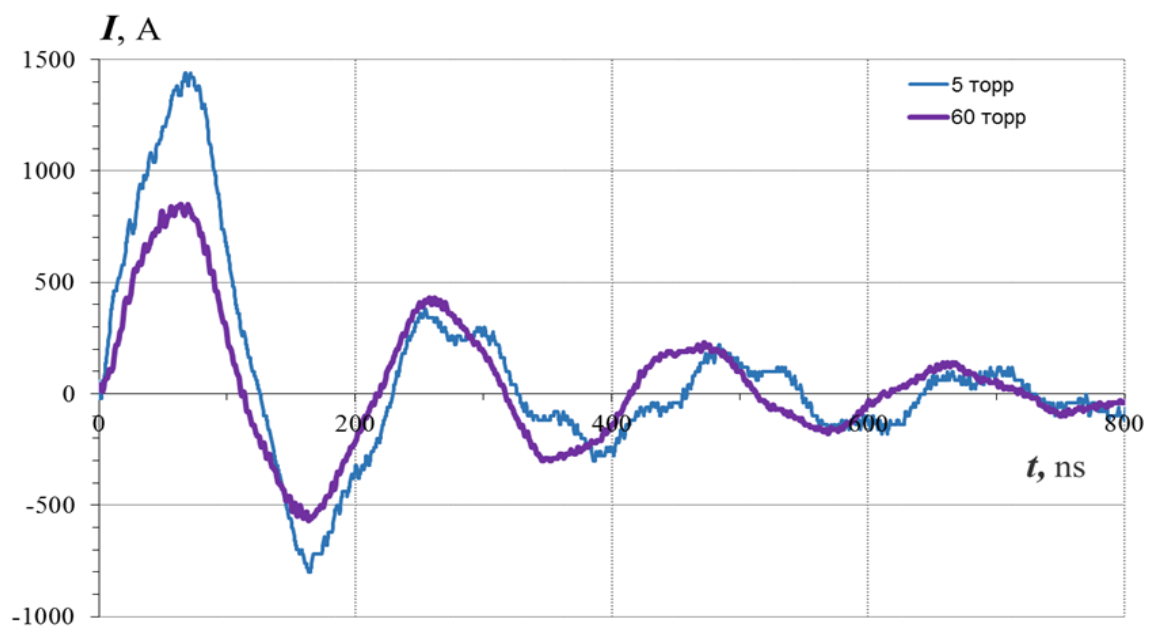

Fig. 3. Current waveforms in still air at a pressure of 5 and 60 torr (density of 0.008 and 0.10 $\mathrm{kg} / \mathrm{m} 3)$.

The analysis of the discharge spectra showed that the main part of the discharge radiation consists of the bands of the second positive nitrogen system ( the wavelength range $280-500 \mathrm{~nm}$ (Fig. 4). In the spectra there are intense lines of atoms of oxygen, nitrogen, hydrogen, formed during the dissociation of molecules. It should be noted that the radiation of the visible part of the spectrum $(400-750 \mathrm{~nm})$ is recorded in the photo images (Fig. 3), the second positive nitrogen system makes a significant contribution. The radiation lifetime of the emitting level of $\mathrm{C}_{3} \mathrm{Pu}$ of molecular nitrogen is about $40 \mathrm{~ns}$. During the passage of the discharge current and radiation from this level, no significant displacement of the structural elements of the high-speed flow occur.

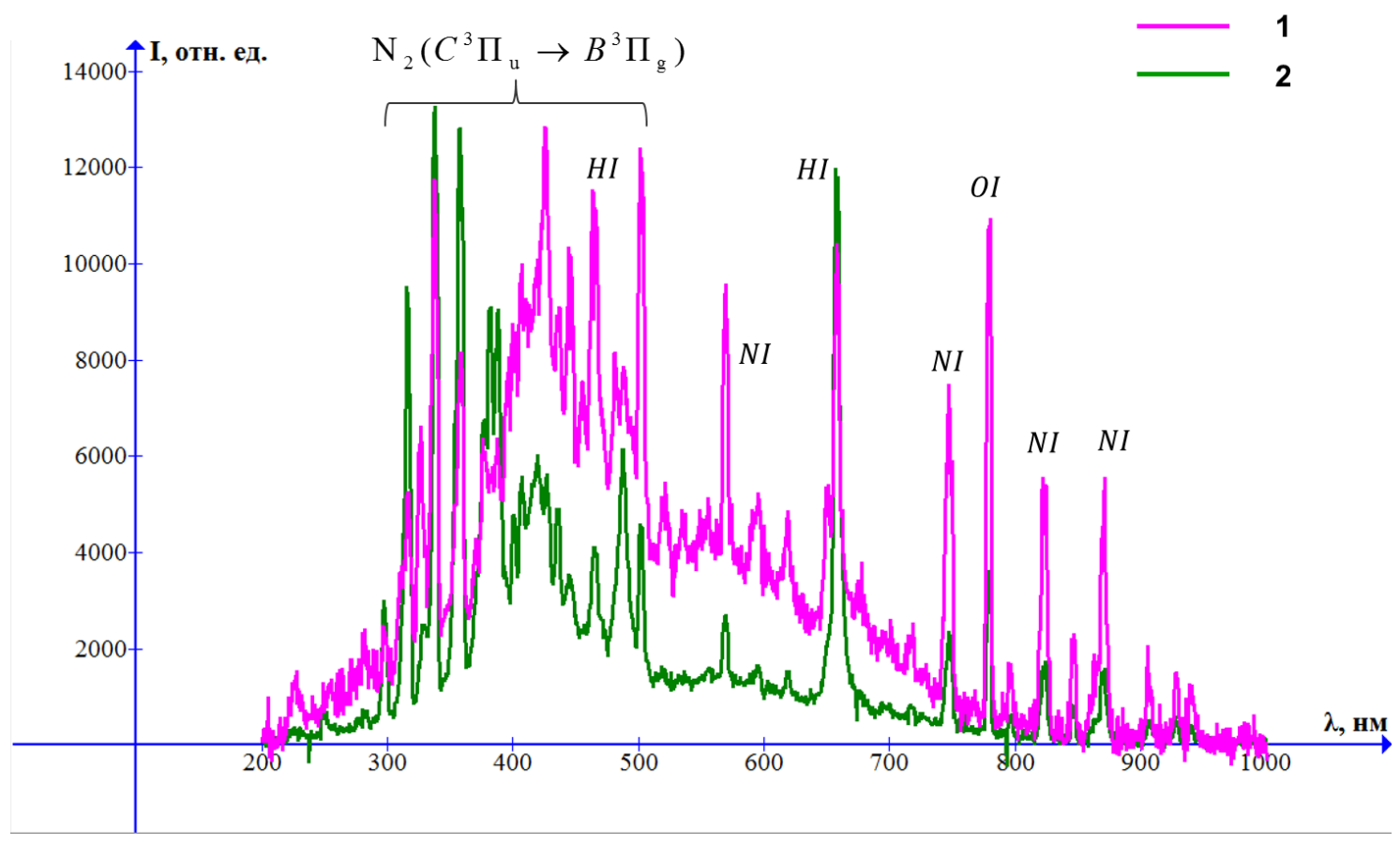

Fig. 4. The emission spectra of the discharge: 1 - in a supersonic flow with a Mach number of $1.38 ; 2$ - in still air; density is $0.10 \mathrm{~kg} / \mathrm{m} 3$. 

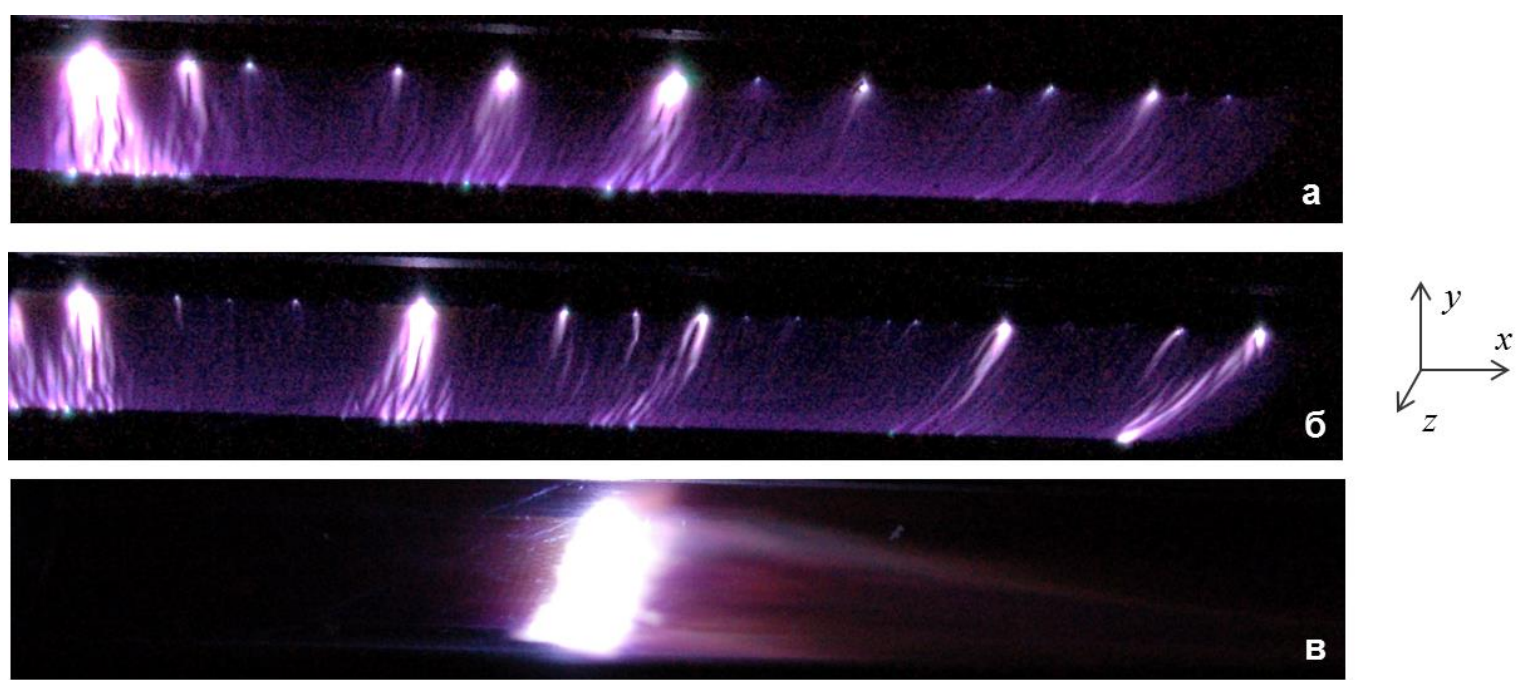

Fig. 5. Photo images of a sliding discharge glow in the still air at a pressure of 33 (a) and 70 Torr (b) (density, respectively, 0.06 and $0.12 \mathrm{~kg} / \mathrm{m} 3$ ) and in a supersonic airflow with an oblique shock wave (c) at a density of $0.12 \mathrm{~kg} / \mathrm{m} 3$ and flow Mach number 1.40 .

\section{DISCHARGE RADIATION UNDER THE INTERACTION OF AN OBLIQUE SHOCK WAVE WITH A BOUNDARY LAYER}

The density distribution in the medium affects the development of nanosecond discharges. The inhomogeneity of the density leads to the inhomogeneity of the reduced electric field $E / N$ and, consequently, the heterogeneity of the local conductivity of the medium (the concentration of electrons). Increased conductivity in areas of low density can lead to a change in the geometry of the current of a pulsed surface sliding discharge [17]. The excitation of electron levels of molecules by electrons leads to an increased intensity of radiation from low-density regions of the flow, hence visualizing them $[12,13,17]$. The spatial distribution of radiation provides information about the instantaneous density distribution in the region of the discharge radiation (in terms of gas dynamics). So, the glow of a pulsed surface sliding discharge in a boundary layer of a uniform supersonic flow clearly visualizes its structure and the region of a laminar - turbulent transition at a certain distance from the shock wave front [13].

The spatial distribution of a sliding discharge radiation in an inhomogeneous supersonic flow in the channel of a shock tube was structurally different at different stages of the formation of a flow with an oblique shock wave. In the case when at the moment of initiation of the discharge the front of a plane shock wave traveling through the channel was inside the discharge region or went a short distance beyond it, the discharge current flowed principally ahead of the wave front. The main radiation of the discharge was concentrated in this region. In fig. 6a, the discharge glow is shown in the form of a U-shaped channel near the shock wave front. At the same time, a weak discharge glow was observed at a distance of 5-6 cm behind the shock wave front, which visualized a zone of low density in the region of interaction of an oblique shock wave with a laminar boundary layer (Fig. 6a). At later stages of the development of the flow, oblique shock wave interacted with the turbulent boundary layer, forming a separation region with a lower density. The discharge glow was concentrated in a single intense channel with a width of less than $10 \mathrm{~mm}$ (Fig. 6b-d). The left boundary of the channel corresponds to a straight line of intersection of the inclined shock wave with the boundary layer, as shown in the photo image obtained by the filter (Fig. 6c). When initiating a discharge after the end of a uniform co-current flow with the Mach number of the flow decreasing, the discharge channel moves upstream, in order to be located closer to the obstacle (Fig. 6g).

An analysis of experimental photo images showed that the structure of the glow of the discharge plasma depends on the flow parameters and is determined by the density distribution in the near-surface flow. The position and geometry of the glow region in the boundary layer 
of the flow were determined on the basis of digital processing of registered photo images from both sides of the discharge chamber. The distance Dk from the beginning of the obstacle on the lower wall of the channel (see Fig. 1 b) to the discharge glow region on the upper wall of the discharge chamber and the length of this region along the flow direction at different stages of the flow (Fig. 7) were measured. These values were measured by scanning images along the OX axis using a Python language program. Fig. $1 \mathrm{~b}$ highlights the boundaries of the regions of the glow in three-color ranges.
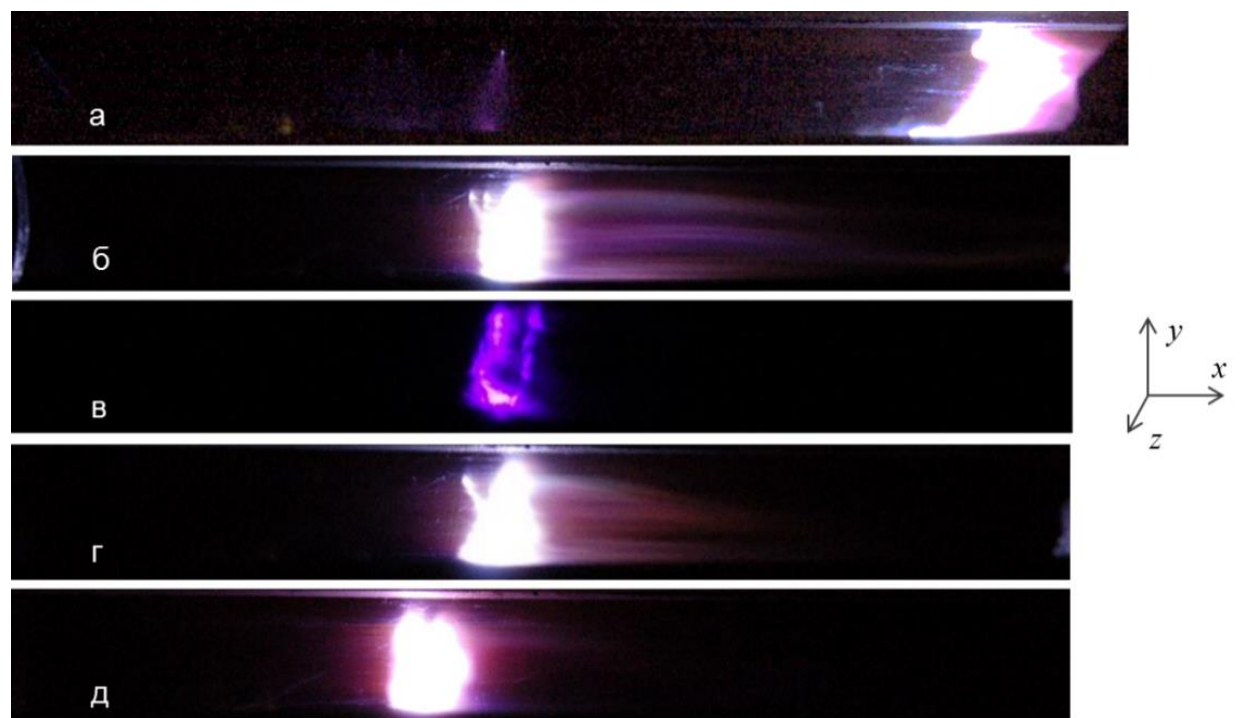

Fig. 6. Photo images of a sliding discharge glow in a flow with an oblique shock wave at discharge initiation times 76 (a), 93 (b), 140 (c), 240 (d), $455 \mu \mathrm{s}$ (e) (from the moment of the shock wave diffraction on an obstacle). The density is $0.13 \mathrm{~kg} / \mathrm{m} 3$, the Mach number of flow is

1.48, the flow is directed from left to right. The image (c) was registered through an optical filter that transmits radiation with a wavelength of $405 \mathrm{~nm}$.

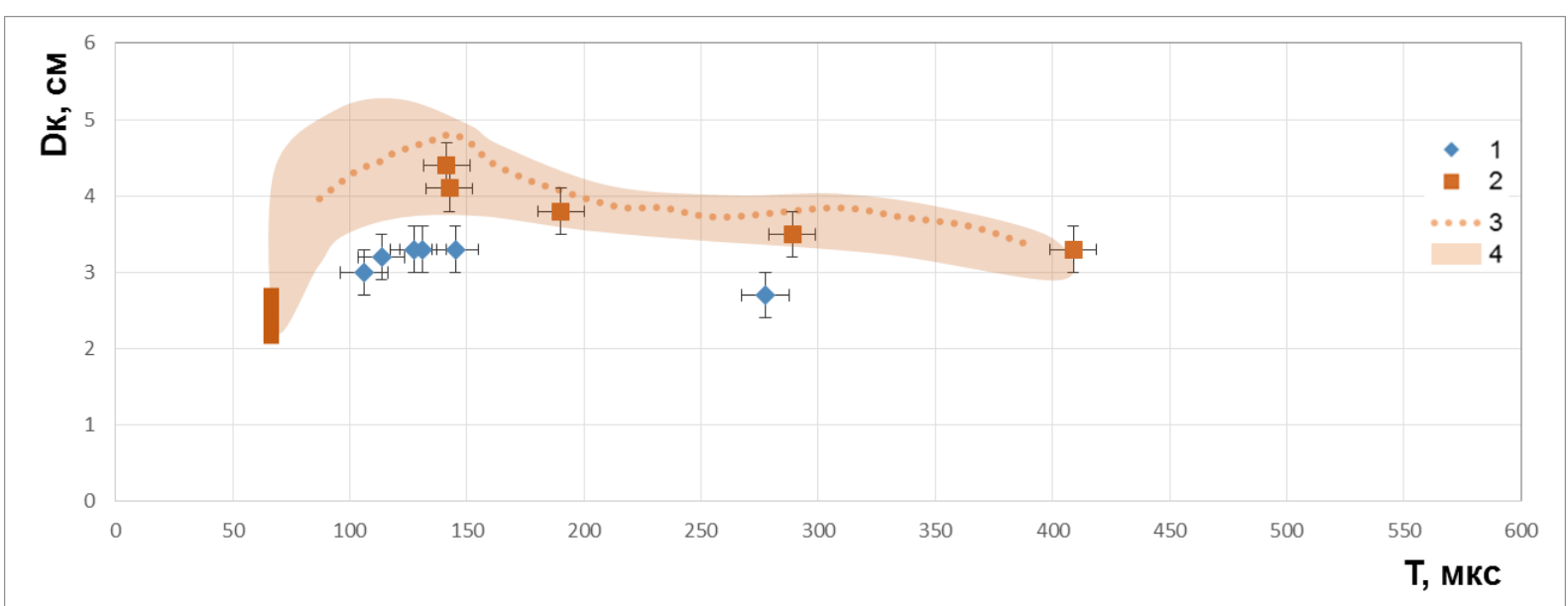

Fig. 7. The coordinate of the region of maximum glow of the discharge channel as a function of time $T$ for flows with Mach numbers 1.36-1.42 (1), 1.45-1.47 (2); the calculated position of the density minimum (3) and the length of the region of low density (4) for the flow Mach number 1.46 . The density is $0.12 \pm 0.01 \mathrm{~kg} / \mathrm{m} 3$. ( $\mathrm{T}=0$ corresponds to the moment when the shock wave contacts the obstacle.)

Dependence between the distance Dk and the time $\mathrm{T}$ for two ranges of flow Mach numbers is shown in fig. 7 ( $\mathrm{T}$ is the time from the beginning of the diffraction of a plane shock wave on an obstacle until the moment of the discharge). The obtained values of Dk are within (27-38) $\pm 2 \mathrm{~mm}$ under the experimental conditions, being in the region of interaction of an oblique shock wave with a boundary layer. The position of the discharge glow area varies 
slightly with time in the range of $150-400 \mu$ s from the beginning of the diffraction of the shock wave. The structure of the discharge glow changes with the increasing distance from the shock front due to a change in the type of interaction of an oblique shock wave with the boundary layer during the transition from the laminar to the turbulent regime. The discharge channel radiation spectrum in this case is characterized by an increased intensity of the continuum and atomic lines of the visible range (Fig. 4), indicating an increased concentration of electrons.

\section{CFD SIMULATION OF THE FLOW IN THE CHANNEL}

The purpose of the numerical simulation was to determine the shock-wave structure of the flow in the channel with an obstacle under experimental conditions and subsequent comparison with the results of the study of the surface sliding discharge in non-uniform supersonic flows.

Mathematical modeling of gas flow in the channel was carried out on the basis of twodimensional Navier-Stokes equations, which describe the unsteady flow of a viscous compressible gas with appropriate initial and boundary conditions and relations of thermal and caloric equations of state [18]. A perfect gas (air) model was used with a constant adiabatic index $(\gamma=1.4)$ and a Prandtl number $\operatorname{Pr}=0.72$. The dependence of the viscosity coefficient on temperature was described by the Sutherland formula [18]. Numerical calculations of flows behind plane shock waves were carried out in a channel with a height of $24 \mathrm{~mm}$ and a length of $216 \mathrm{~mm}$ under experimental conditions. In the numerical simulation a plane shock wave diffracted on a 2'6 mm obstacle located on the lower wall of the channel. Fig. 8 shows the successive stages of the interaction of a plane shock wave with an obstacle. After diffraction of a shock wave for $210-230 \mu \mathrm{s}$, a quasi-stationary flow around the obstacle with a supersonic flow with an inclined shock wave is established in the channel. Boundary layers are formed on the upper and lower walls, disturbed by the interaction with oblique shock waves. On the calculated flow fields, the dynamic color scale is used thus the maximum value of the given value corresponds to the red color, the minimum corresponds to the blue.

In the experiments the discharge was initiated on the upper wall of the channel in the region corresponding to the dimensionless coordinates of the computational domain from 1.9 to 6.0 (dimensions are related to the $24 \mathrm{~mm}$ channel height). In this area the oblique shock from the obstacle interacts with the boundary layer on the upper wall of the channel (Fig. 8). This interaction leads to the formation of a region of low density or flow separation region (Fig. 9), located at a distance of $38-48 \mathrm{~mm}$ from the leading edge of the obstacle at steady state. The length of the low-density region (along the flow direction) was 5-15 $\mathrm{mm}$, depending on the time after the diffraction of the initial shock wave on the obstacle. The region of interaction of an oblique shock wave with a boundary layer on the upper wall of the channel is shown on an enlarged scale in Fig. 9 b. While conducting the calculations the thickness of the undisturbed boundary layer did not exceed $0.2 \mathrm{~mm}$, and the separation zone was no more than $1.5 \mathrm{~mm}$ thick. The minimum density in this area was $38-62 \%$ of the incident flow density. 

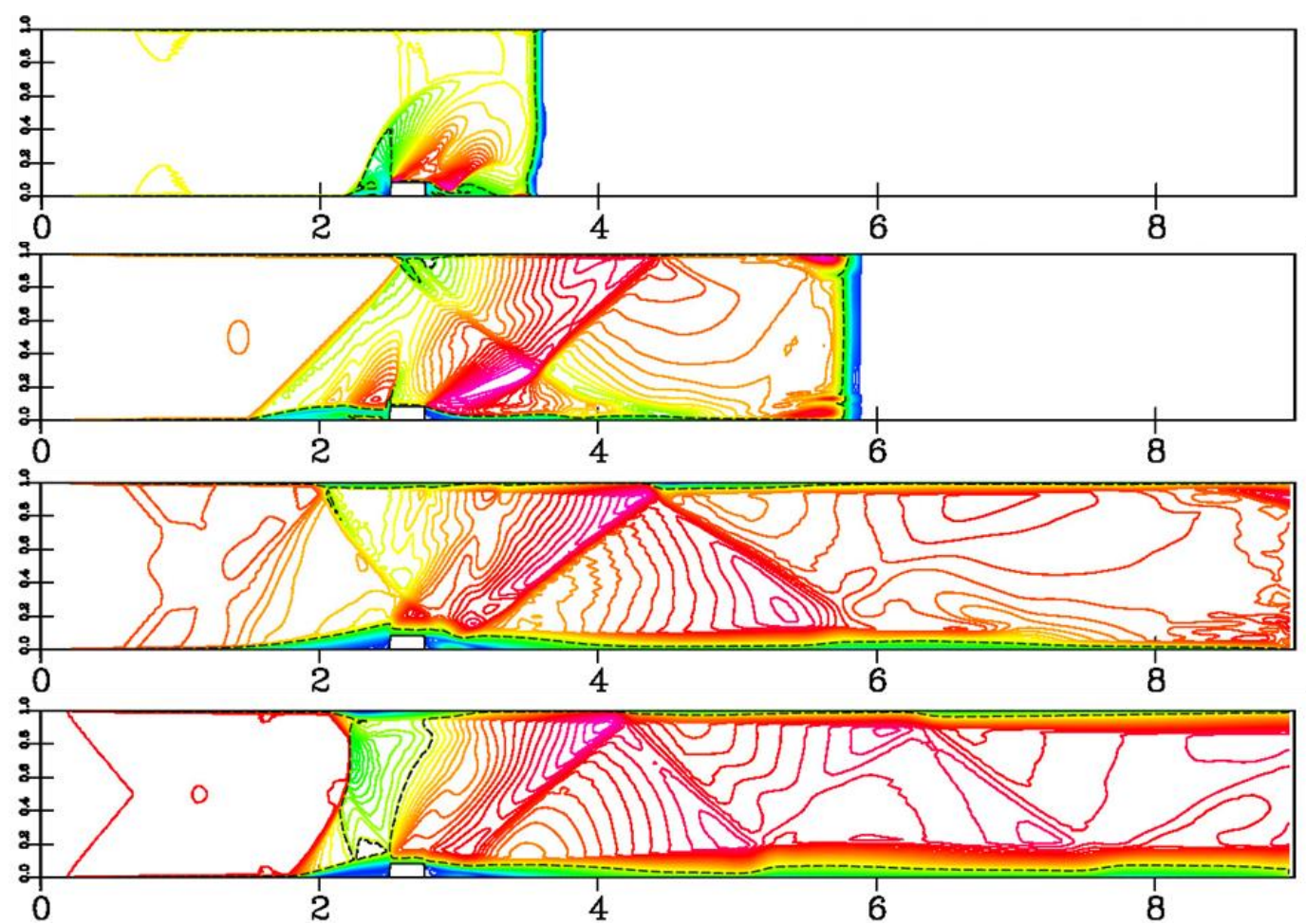

Fig. 8. The field of the local Mach number in establishing the flow around an obstacle with a flow behind the shock wave with a Mach number of 3.45: the time from the moment the shock wave contacts the obstacle $30,80,180,310 \mu$ s (from top to bottom).

a)
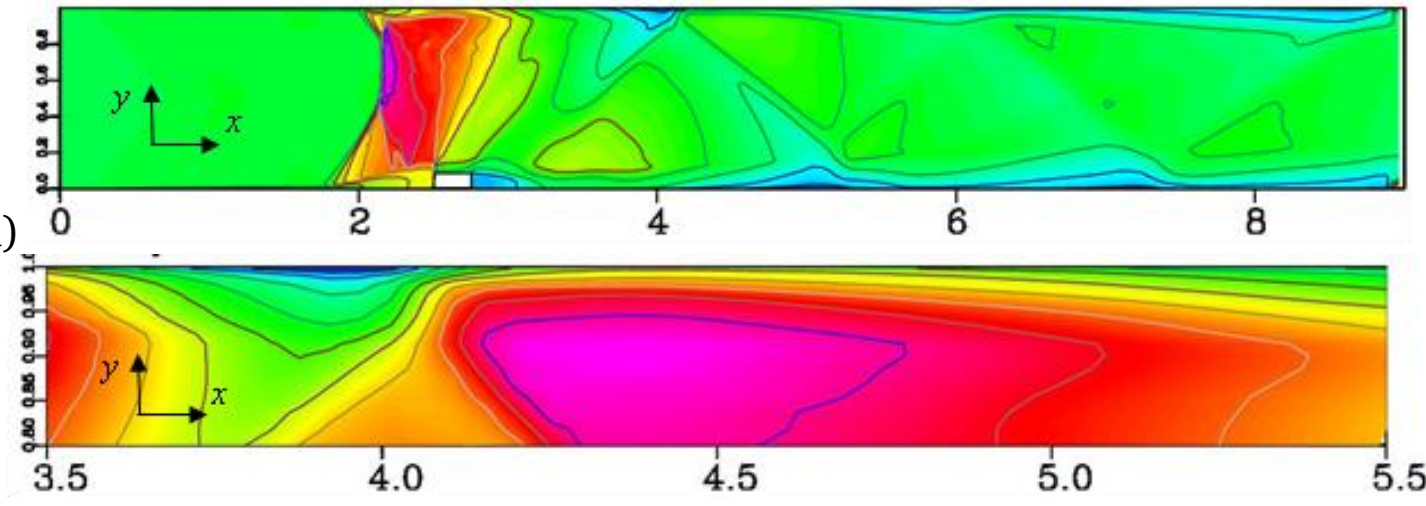

Fig. 9. The calculated density field at the quasi-stationary stage of the flow (a), an enlarged fragment of the region of interaction of an oblique shock wave with the boundary layer on the upper wall of the channel (b). The Mach number of the initial shock wave is 3.45, the time from the moment the shock wave contacts the obstacle is $310 \mu \mathrm{s}$.

The dependence of the calculated position of a low-density region on time is shown in Fig. 7. The length and position of this region depends on time: first, it forms as an extended zone within 22-43 $\mathrm{mm}$ from an obstacle, then shifts downstream in the process of flow establishing, and is located at a distance of $36-42 \mathrm{~mm}$ from an obstacle at the steady-state flow (150-350 $\mu$ s from the diffraction moment). According to the fig. 7 the experimental dependence of the location of the discharge glow region on time clearly correlates with the dependence of the position of the low-density region on time in the calculated data. Thus, a comparison of the experimental data with the calculated indicates that the glow geometry of a pulsed surface sliding discharge coincides with the geometry of a low-density zone such as its location and length. This allows to visualize the structure of the boundary layer area under study based on the registration of discharge radiation developing inside the boundary layer. The nanosecond discharge duration, which is much shorter than the characteristic gas-dynamic times, makes it possible to investigate non-stationary flows with shock waves. 


\section{CONCLUSION}

The effect of inhomogeneous supersonic flow in a channel behind shock waves with Mach numbers 2.8-4.2 on the spatial structure of radiation of a surface sliding discharge of nanosecond duration is studied experimentally. An analysis of the discharge glow in the region of the interaction of an oblique shock wave with the boundary layer, including the discharge in the form of an intense channel in the zone of separation of the boundary layer is carried out. It is shown that the glow of a pulsed surface sliding discharge allows visualizing the region of interaction of an oblique shock wave with a boundary layer at different stages of an unsteady flow. Registration of discharge radiation at different angles can provide information about the three-dimensional structure of the boundary layer zone. The advantages of the visualization method on the base of the registration of radiation from the discharge plasma are that the plasma locates directly into the boundary layer, and the short discharge duration, which makes it possible to investigate non-stationary flows.

The work was supported by RFBR grant 19-08-00661.

\section{REFERENCES}

1. Zheltovodov A. A. Properties of two- and three-dimensional separation flows at supersonic velocities // Fluid Dynamics. 1979. V. 14. No 3. P. 357-364.

2. Zubin M. A., Ostapenko N. A. Structure of flow in the separation region resulting from interaction of a normal shock wave with a boundary layer in a corner // Fluid Dynamics. 1979. V. 14. No 3. P. 365-371.

3. Kubota H., Stollery J. An experimental study of the interaction between a glancing shockwave and a turbulent boundary layer // J. Fluid Mech. 1982. 116-431-58.

4. Dupont P., Haddad C., Debiève J.-F. Space and time organization in a shockinduced separated boundary layer. J. Fluid Mech. 2006. 559. P. 255-277.

5. Borovoy V.Ya., Mosharov V.N, Noev A.Yu., Radchenko V.N. Laminar-turbulent flow over wedges mounted on sharp and blunt plates // Fluid Dynamics. 2009. V. 44. No 3. P. 382-396.

6. Hadjadj A., Perrot Y., Verma S. Numerical study of shock/boundary layer interaction in supersonic overexpanded nozzles // Aerosp. Sci. Technol. 2015. V. 42. P. 158-168.

7. Bosnyakov S.M., Babulin A.A., Vlasenko V.V., Engulatova M.F., Matyash S.V, Mikhaylov S.V. On the accuracy of numerical simulation of the boundary layer separation on a finite-width wedge. // Mathematical Models and Computer Simulations. 2016. V. 8. No 3. P. 238-248.

8. Bayoda D., Benard N., and Moreau E. Nanosecond pulsed sliding dielectric barrier discharge plasma actuator for airflow control: Electrical, optical, and mechanical characteristics // J. Appl. Phys. 2015. V. 118. 063301.

9. Mursenkova I.V., Znamenskaya I.A. and Lutsky A.E. Influence of shock waves from plasma actuators on transonic and supersonic airflow // J. Phys. D: Appl. Phys. 2018. V. 51. N 5. 105201.

10. Leger L., Sellam M., Barbosa E. and Depussay E. Visualization by discharge illumination technique and modification by plasma actuator of rarefied Mach 2 airflow around a cylinder // Meas. Sci. Technol. 2013. V. 24. N 6. 065401

11. Ju Y. and Sun W. Plasma assisted combustion: Dynamics and chemistry // Progress in Energy and Combustion Science. 2015. V. 48. P. 21-83.

12. Znamenskaya I.A., Latfullin D.F. and Mursenkova I.V. Laminar-Turbulent Transition in a Supersonic Boundary Layer during Initiation of a Pulsed Surface Discharge. // Technical Physics Letters. 2008. V. 34. No. 8. P. 668-670.

13. Znamenskaya I., Mursenkova I., Kuli-Zade T., Kolycheva A. Vizualization of $3 \mathrm{D}$ non-stationary flow in shock tube using nanosecond volume discharge // Shock Waves. 2009. P. 533-538. 
14. Nishio M., Sezaki S., Nakamura H. Visualization of flow structure around a hypersonic re-entry capsule using the electrical discharge method // Journal of Visualization. 2004. V. 7. N 2. P. 151-158.

15. Bazhenova T.V., Gvozdeva L.G. Nestacionarnye vzaimodejstviya udarnyh voln. M., 1977. 274 p. [In Russian]

16. Raizer Yu. P. Gas Discharge Physics (Springer, Berlin, 1991)

17. Mursenkova I.V., Sazonov A.S., and Liao Yu. The Effect of Pulsed Sliding Surface Discharges on Supersonic Airflow past a Thin Wedge in Shock Tube. // Technical Physics Letters, 2018 V. 44. No. 2. P. 157-159.

18. Glushko G.S., Ivanov I.E., Kryukov I.A. Computational method for turbulent supersonic flows // Mathematical Models and Computer Simulations. 2010. V. 2. No 4. P. 407422. 